\title{
Exercise-based interventions for Indigenous adults with chronic lung disease in Australia, Canada, New Zealand, and USA: a systematic review
}

\author{
David P. Meharg ${ }^{1,2} \wedge$, Kylie Gwynne ${ }^{2,3} \wedge$, John Gilroy ${ }^{1 \wedge}$, Jennifer A. Alison ${ }^{1,4} \wedge$ \\ ${ }^{1}$ Faculty of Medicine and Health, Sydney School of Health Sciences, The University of Sydney, Sydney, Australia; ${ }^{2}$ Poche Centre for Indigenous \\ Health, The University of Sydney, Sydney, Australia; ${ }^{3}$ Faculty of Medicine, Health and Human Sciences, Macquarie University, Sydney, Australia; \\ ${ }^{4}$ Sydney Local Health District, Sydney, Australia \\ Contributions: (I) Conception and design: DP Meharg, JA Alison, K Gwynne; (II) Administrative support: DP Meharg, JA Alison; (III) Provision of \\ study materials or patients: DP Meharg, JA Alison; (IV) Collection and assembly of data: DP Meharg, JA Alison; (V) Data analysis and interpretation: \\ DP Meharg, JA Alison; (VI) Manuscript writing: All authors; (VII) Final approval of manuscript: All authors. \\ Correspondence to: David P. Meharg. The University of Sydney, R.C Mills Building (A26), Fisher Road, Camperdown, NSW, 2006, Australia. \\ Email: David.Meharg@sydney.edu.au.
}

\begin{abstract}
Indigenous peoples in Australia, New Zealand, Canada, and the United States of America (USA) have a higher burden of chronic lung disease than non-Indigenous people. Exercised-based interventions, such as pulmonary rehabilitation, are highly effective to manage chronic lung disease. The outcomes of these interventions for Indigenous people require evaluation. The aim of this review was to critically appraise the literature on the impact of exercise-based interventions on quality of life, exercise capacity and health care utilisation in Indigenous adults with chronic lung disease in Australia, New Zealand, Canada, and USA. The Cochrane Library, Medline, Embase, CINAHL, Scopus, Psychinfo, APAIS-Aboriginal Health and PEDro databases were searched for peer-reviewed and grey literature that evaluated exercise-based interventions, such as pulmonary rehabilitation for Indigenous adults with chronic lung disease in Australia, New Zealand, Canada, and USA. Two authors independently screened and reviewed titles and abstract and full texts of potentially eligible studies for inclusion. An Indigenous decolonisation methodological framework was also applied to evaluate Indigenous governance, involvement, and engagement in the studies. A total of 3,598 records were screened, nine full papers were reviewed, and one was study included, which was a cardiopulmonary rehabilitation program for Indigenous people in Australia. Participants with chronic respiratory or heart disease significantly improved functional exercise capacity and quality of life [six-minute walk distance mean change (95\% CI) 79 metres (47 to 111); Chronic Respiratory Questionnaire Dyspnoea 0.9 points (0.2 to 1.5$)$ ]. Several items of the decolonisation framework were addressed. Only one study was able to be included in the review, highlighting the paucity of research about culturally safe exercise-based interventions for Indigenous adults with chronic lung disease. There is a need for further research with strong Indigenous governance, involvement, and engagement.
\end{abstract}

Keywords: Indigenous; aboriginal; first nations; chronic obstructive pulmonary disease; pulmonary rehabilitation

Submitted May 18, 2020. Accepted for publication Oct 14, 2020.

doi: $10.21037 /$ jtd-20-1904

View this article at: http://dx.doi.org/10.21037/jtd-20-1904

$\wedge$ ORCID of David P. Meharg: 0000-0003-1231-5854; Kylie Gwynne: 0000-0002-6897-4528; John Gilroy: 0000-0002-7934-8485; Jennifer A. Alison: 0000-0002-2011-4756. 


\section{Introduction}

Indigenous peoples in high-income countries where colonisation has occurred, such as Australia, New Zealand, Canada and the United States of America (USA), have poorer health outcomes and shorter life expectancy when compared to non-Indigenous peoples $(1,2)$. These Indigenous peoples include Aboriginal and Torres Strait Islander peoples in Australia, Māori in New Zealand, First Nations Indigenous which includes First Nations, Innu/ Inuit and Metis people in Canada, and American Indian and Alaska Native tribes in the USA (2). Chronic lung diseases, such as chronic obstructive pulmonary disease (COPD) and bronchiectasis, have a higher prevalence (3-7), hospitalisation and mortality rate in these Indigenous populations compared to non-Indigenous populations in these countries (3-10). The relative prevalence of interstitial lung disease in Indigenous populations compared to nonIndigenous populations is less well known, some studies suggest higher rates in Indigenous populations (11).

Higher smoking rates partly contribute to this burden of disease (12), but is compounded by reduced access, availability and utilisation of appropriate health care and health education that could ameliorate the effects of chronic lung disease in Indigenous populations (6,13-15).

The economic and social burden of chronic lung disease, especially COPD is significant (16). Australia, New Zealand and Canada spend billions of dollars annually on direct and indirect costs (17-19), though the USA have the highest estimated direct cost totalling US\$32 billion and indirect costs of \$20.4 billion annually for COPD (20).

National clinical guidelines for the management of COPD in Australia, New Zealand, Canada, and USA strongly advocate that pulmonary rehabilitation should be available for people with COPD $(8,21,22)$. In addition, pulmonary rehabilitation guidelines recommend that pulmonary rehabilitation should be available to people with other chronic lung diseases, such as bronchiectasis and interstitial lung disease (ILD) (23). Pulmonary rehabilitation is the most effective non-pharmacological intervention to improve health-related quality of life and function, reduce symptoms of breathlessness and fatigue (24), and help prevent hospital admissions in people with COPD (25) and has been shown to improve similar outcomes for people with bronchiectasis $(26,27)$. As such, pulmonary rehabilitation, a supervised exercise-based intervention with health education (28) is recommended as an important component of management of people with COPD (29) and other chronic lung diseases, such as bronchiectasis and ILD (23). However, current guidelines do not offer specific cultural advice to clinicians to enable Indigenous peoples, their families, and communities to better manage these conditions. Further, only one pulmonary rehabilitation guideline has highlighted the inequality of access of Indigenous peoples to culturally safe programs (23).

Cultural safety is an Indigenous-led model of care that aims to redress power imbalances between health care providers and consumers, and in which clinicians undertake cultural self-reflection, bringing awareness to power relationships to decolonise and improve health practices (30). Indigenous peoples are less likely to access health care than non-Indigenous peoples for reasons including a lack of culturally safe services, low health literacy (31), living remotely and/or on reservations, and in some countries, due to inadequate health insurance coverage (32). When Indigenous peoples eventually utilise health care, they report additional barriers such as racism and receiving inappropriate care (33), leading to feelings of fear, distrust and alienation (34). Provision of culturally safe care is a key factor for Indigenous people to access health services, and the ability of services to achieve positive patient outcomes (35-37), including the uptake and completion of pulmonary rehabilitation (38).

Although exercise-based interventions, such as pulmonary rehabilitation programs are potentially available to Indigenous people through mainstream hospital-based programs, uptake is low (39). Barriers for the general population to attend pulmonary rehabilitation have been reported as lack of transport, difficult locations to access, inconvenient timing, disruption of usual routine and lack of encouragement by health professionals (40). Additional barriers for Indigenous people to attend mainstream pulmonary rehabilitation programs relate to the lack of attention to cultural needs and the perception that programs are unwelcoming or unsafe (38). This can be further compounded by the dominant Western medical model that focuses on individual well-being with a disease-specific approach, which can conflict with the holistic construct that incorporates the social, cultural and community well-being common in Indigenous cultures (41).

Indigenous community controlled health services have improved access to and delivery of culturally safe health care, enhancing Indigenous peoples health outcomes (42). Such services play a key role reversing the power imbalances and inequities caused by colonisation, and enable self-determination of Indigenous peoples within 
the Australian, New Zealand, Canadian and USA health care systems (43-46). These services have also increased access to and availability of chronic disease prevention and management services (43), with reports from health consumers that these services are equal to or better than general practice (42). Features of these service providers include strong collaborative relationships with Indigenous communities to develop services incorporating cultural values and perspectives to prevent chronic conditions (44). Gaps in service provision are addressed through effective partnerships that promote community well-being and innovative service delivery reforms (47). There is growing evidence that Indigenous governance of health programs are leading to better approaches to health problems (45). Since there is a higher incidence of chronic lung disease, especially COPD in Indigenous populations and pulmonary rehabilitation is a key component of the management of COPD, knowledge of the availability of exercise-based training, such as pulmonary rehabilitation programs and their outcomes in Indigenous peoples is important.

The aim of this systematic review was to critically appraise the literature on the impact of exercise-based interventions or pulmonary rehabilitation on quality of life, exercise capacity and health care utilisation in Indigenous adults with chronic lung disease in Australia, New Zealand, Canada, and USA. We present the following article in accordance with the PRIMSA reporting checklist (available at http://dx.doi.org/10.21037/jtd-20-1904).

\section{Methods}

The PROSPERO database and Cochrane Library were searched for systematic reviews on exercise-based interventions or pulmonary rehabilitation programs for Indigenous peoples with chronic lung disease in Australia, New Zealand, Canada, and USA. After determining no such reviews were published or in progress, the current review was designed in accordance with the Preferred Reporting Items for Systematic Reviews and Meta-Analyses (PRISMA) statement and checklist for systematic reviews (48) and registered prospectively with PROSPERO (registration number: CRD42019111405). The search strategy was designed in consultation with a university health sciences librarian.

The Cochrane Library, Medline, Embase, CINAHL, Scopus, Psychinfo, APAIS-Aboriginal Health and PEDro were searched from their respective start dates until November 2019 for exercise training or pulmonary rehabilitation interventions for Indigenous people with chronic lung disease in Australia, New Zealand, Canada, or USA. Search terms for populations were 'Indigenous' OR 'First nation* OR 'Aborigin*' OR 'North American Indian*' OR 'Inuit*' OR 'Torres Strait Islander* OR 'Maori' OR 'Rural population' OR 'Rural environments' and were combined with 'Chronic Obstructive Pulmonary Disease' OR 'COPD' OR 'Lung Disease*'OR 'Pulmonary Disease*' OR 'Pulmonary Emphysema' OR 'DYSPNEA' OR 'Respiratory Tract Disorders' OR 'respiratory tract infection*' OR 'respiratory tract disease' OR 'Interstitial lung disease*' OR 'Emphysema' OR 'Obstructive lung disease*' OR 'interstitial lung disease' OR 'ILD' OR 'lung emphysema' OR 'paroxysmal dyspnea' OR 'breathing disorder' OR 'respiration disorder*' OR 'Paroxysmal' and other terms 'indigenous health care' OR 'indigenous people' OR 'Aborigine' OR 'Health Services'. No restriction on language or publication years were applied. Forward and backward citation tracking from identified papers was undertaken. Government websites and databases were searched for grey literature. Experts in the field, based in Australia, New Zealand, Canada, and USA were also contacted to provide information on additional publications.

\section{Study inclusion and exclusion criteria}

To be included in the review, studies had to evaluate an intervention with the primary focus of exercise training and/or pulmonary rehabilitation for Indigenous adults, 18 years of age and older with chronic lung disease in Australia, Canada, New Zealand or USA, and report any of the following outcomes: health-related quality of life, exercise capacity, health care utilisation or mortality. The definition of pulmonary rehabilitation used for inclusion of studies was: 'any in-patient, out-patient, community-based or home-based rehabilitation programme of at least four weeks duration that included exercise therapy with or without any form of education and/or psychological support delivered to patients with exercise limitation attributed to COPD' (24) or other chronic lung disease. Controlled and uncontrolled studies were eligible for inclusion. Studies had to include Indigenous peoples with chronic lung disease and provide outcomes assessed using psychosocial, behavioural, or clinical measures. Studies were excluded if they consisted of populations other than those stated in the inclusion criteria, where Indigenous and non-Indigenous disaggregated data was not provided or studies that lacked an exercise-based intervention or outcomes related to the study aim. 


\section{Data extraction and assessment}

Articles were scanned for inclusion based on title and abstract by two authors (DP Meharg and JA Alison). Differences were resolved through discussion and consensus. Data from full texts were extracted independently by two reviewers (DP Meharg and JA Alison). Information of interest included study population, recruitment strategy, study setting, details of the intervention, exercise capacity, health-related quality of life and health care utilisation, as well as Indigenous involvement in the study design, community engagement and governance guided by a decolonisation methodological framework. Colonisation has resulted in power imbalances between non-Indigenous and Indigenous peoples, particularly with how research is undertaken. A decolonisation methodological framework explores how Indigenous knowledge and values were reflected in the research methods of studies (49). While not included in the original prospectively registered protocol, the decolonisation methodological framework was used to evaluate included studies for Indigenous involvement, Indigenous community engagement and Indigenous governance. A decolonisation lens has been used in similar work $(1,49-51)$ and was modified by the Indigenous scholars on the research team (DP Meharg and J Gilroy) to address the following questions: 'How did the researchers include Indigenous voices?', 'What model of research governance was adopted that empowered Indigenous people in the research'?, 'What was being said about Indigenous people?', 'Who was speaking?', and 'Where were the research findings published?

The quality of included studies was assessed independently by two authors (DP Meharg and JA Alison) using the Effective Public Health Practice Project Quality Assessment Tool (52). This tool was chosen instead of the GRADE system that was originally detailed in the prospectively registered protocol, as it has been used in several Indigenous systematic reviews (53-55), and is appropriate for a range of controlled and uncontrolled study designs $(56,57)$, and has demonstrated fair inter-rater reliability (58). Using this tool, studies were evaluated for selection bias, study design, confounders, blinding, data collection methods, withdrawals, and dropouts. Each category was given an individual rating and studies were given an overall rating of strong, moderate, or weak for quality. Narrative synthesis was guided by the Cochrane Consumers and Communication Review Group (59).

\section{Results}

Search results and screening outcome are presented in a flow-diagram (Figure 1). Through database searching, 5,696 records were retrieved. Two additional records were identified from backward and forward citation tracking, totalling 5,698 records. No articles were located in the grey literature. After removing duplicates, the titles, and abstracts of 3,598 records were screened. Nine full papers were reviewed. Eight studies were excluded based on full text review, as they did not include either an intervention, Indigenous peoples, or present data specifically on participants with chronic lung disease. One interventional study met the eligibility criteria (39). Based on this result, a narrative review was performed. The data collection methods were rated as strong, but the inability for blinding of the exercise intervention, lack of randomisation and selection bias, resulted in the overall quality rating of the study being weak (60) (Table 1). The decolonisation methodological framework review showed that there was the use of Indigenous research governance and involvement with the use of Indigenous voices. Aspects of Indigenous engagement were present, though the study did not report community engagement to support pre-study design or study implementation (Table 2).

The population in the included study was Indigenous adults in Australia diagnosed with COPD, ischaemic heart disease or heart failure, or at high risk of developing these conditions due to at least two cardiovascular risk factors (smoking, obesity, hypertension, diabetes, or dyslipidaemia). The rehabilitation program was delivered between October 2011 and February 2013 by an Aboriginal Community Controlled Health Service based in two major cities in Tasmania, Australia.

The cardiopulmonary rehabilitation program consisted of 16 supervised exercise sessions, for 1 hour, twice weekly for 8 weeks. The exercise sessions were individually prescribed and progressed (based on levels of breathlessness and perceived exertion) by a physiotherapist or exercise physiologist and included stationary cycling, steps, weights and, in some instances, outside walking. Group education sessions were conducted for an hour, once a week. Topics included self-management, healthy eating, smoking cessation, medication use, stress management and psychological well-being. Outcome measures were functional exercise capacity [measured by the 6-minute walk test (6MWT) and the incremental shuttle walk test (ISWT)], the Timed Up and Go (TUG) test, dyspnoea and fatigue 


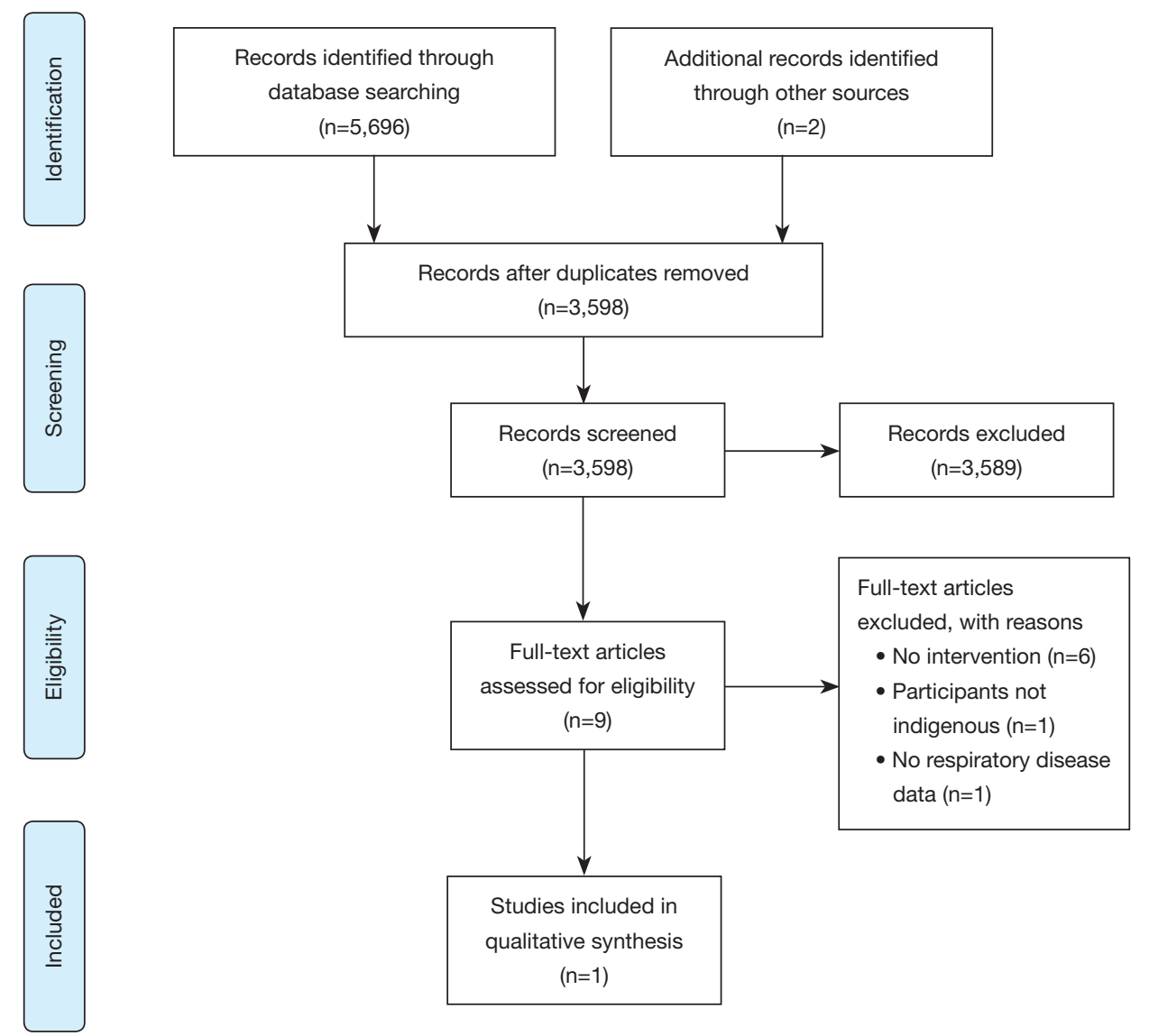

Figure 1 PRISMA flow diagram.

[measured by the Chronic Respiratory Questionnaire (CRQ)], and generic quality of life [measured by the Medical Outcomes Short Form (SF36)].

In total, 92 participants attended at least one exercise session within a program [mean (SD) attendance 11.5 (3.1) sessions] and $0-8$ education sessions [mean attendance 5.4 (2.4) sessions]. Thirty-nine percent of the participants $(n=36)$ had a diagnosis of chronic cardiovascular or respiratory disease and attended at least one of the 8-week programs. There was no further breakdown of numbers of participants with COPD or subgroup analyses of those with COPD alone. Change data from those with a chronic cardiac or respiratory condition showed clinically significant improvements in exercise capacity [mean difference $(95 \%$ CI) 6MWT 79 metres (47 to 111), n=22; ISWT 97 metres (40 to 153 ), $n=19$ ], however it was not clear whether two $6 \mathrm{MWTs}$ and two ISWTs were performed at program entry. As there is a learning effect in these tests (61), the change may have overestimated the real effect of the rehabilitation program. There was a clinically significant improvement in dyspnoea [CRQ Dyspnoea 0.9 points $(0.2$ to 1.5$), \mathrm{n}=20$ ] but not fatigue [CRQ Fatigue 0.4 points ( -0.4 to 1.1$), n=19$ ]. Quality of life scores improved significantly in the following domains of the SF36: Vitality 12.1 (0.7 to 23.5), n=35; Role emotional 16.0 (1.6 to 30.3 ), $n=36$; Mental health 14.3 (7.2 to 21.4$), \mathrm{n}=35$.

Fifty-five percent of participants and staff members completed program evaluations forms. Results revealed participants encouraged others in the community to join the program. Further, a crucial factor for program participants to stay engaged with the study was the encouragement received from other participants and staff, as well as small group team environments and existing knowledge of other participants as local Indigenous community members. Staff completed evaluation forms and a semi-structured interview, which reported that witnessing participants' optimism, health improvements and the comradeship between staff and participants were meaningful outcomes. Aboriginal 
Table 1 Quality rating score of the included study*

\begin{tabular}{|c|c|c|c|c|c|c|c|}
\hline Author & Selection bias & Study design & Confounders & Blinding & $\begin{array}{l}\text { Data collection } \\
\text { methods }\end{array}$ & $\begin{array}{l}\text { Withdrawals and } \\
\text { dropouts }\end{array}$ & $\begin{array}{l}\text { Overall } \\
\text { rating }\end{array}$ \\
\hline $\begin{array}{l}\text { Davey et al., } \\
2014\end{array}$ & Weak & Moderate & Weak & Weak & Strong & Moderate & Weak \\
\hline
\end{tabular}

*, based on Quality Assessment Tool for Quantitative Studies (60).

Table 2 Decolonisation methodological framework

\begin{tabular}{|c|c|}
\hline Author and date & Davey et al., 2014 \\
\hline Aim of study & $\begin{array}{l}\text { To evaluate the provision of culturally accessible cardiac and pulmonary rehabilitation to increase } \\
\text { participation and improve health outcomes for people with established cardiac or pulmonary disease, and } \\
\text { reduce risk factors for people with risk factors only. }\end{array}$ \\
\hline $\begin{array}{l}\text { What is being said about } \\
\text { indigenous people? }\end{array}$ & $\begin{array}{l}\text { Indigenous peoples have high COPD prevalence and rates of hospitalisation. Importance of culturally safe } \\
\text { cardiopulmonary rehabilitation program designed and delivered by an Aboriginal community-controlled } \\
\text { service for the Indigenous community. }\end{array}$ \\
\hline Indigenous specific research & Yes. \\
\hline $\begin{array}{l}\text { Indigenous research } \\
\text { governance }\end{array}$ & $\begin{array}{l}\text { Yes. Strong Indigenous governance model. Governance by Tasmanian Aboriginal Centre. Ethics approval } \\
\text { by the State Committee of the Tasmanian Aboriginal Centre. }\end{array}$ \\
\hline Indigenous engagement & $\begin{array}{l}\text { Partial. Indigenous study participants provided feedback via written evaluation forms. Staff who } \\
\text { participated in the study provided feedback via interviews and written evaluations forms. Study did not } \\
\text { report Indigenous community engagement to support pre-study design or implementation. }\end{array}$ \\
\hline Indigenous publication & No. Published in a non-Indigenous specific peer reviewed journal (BMC Health Services Research). \\
\hline Data collection methods & $\begin{array}{l}\text { Participants completed exercise capacity assessments, functional capacity assessments and quality of } \\
\text { life questionnaires as well as a program evaluation form. Staff completed semi-structured interviews and } \\
\text { provided written feedback. }\end{array}$ \\
\hline Analytic framework & $\begin{array}{l}\text { Quantitative analysis on participation and rehabilitation outcomes. Thematic analysis of qualitative } \\
\text { information from participants and staff. }\end{array}$ \\
\hline
\end{tabular}

Health Workers (AHWs) stated it was pleasing to be seeing improvements in participants, as well as achieving personal and professional benefits by developing new skills. The evaluation revealed that a strength of the program was designing a specific program for Indigenous peoples, with individually designed exercise training, linked to goals.

\section{Discussion}

The purpose of this study was to critically review the literature on the impact of exercised-based interventions, such as pulmonary rehabilitation for Indigenous adults with chronic lung disease in Australia, New Zealand, Canada, and
USA. To the authors' knowledge, this is the first published literature review on this topic. Australia, New Zealand, Canada, and USA were selected as they share similarities such as the historical and ongoing impact of colonisation and an over-representation of Indigenous peoples with chronic lung disease in comparison with non-Indigenous peoples (3-6,8). Despite a comprehensive literature search, only one study met the inclusion criteria. It is possible some interventions were not included due to no formal publications. Given the higher prevalence of chronic lung disease in Indigenous peoples in the targeted countries, and the acknowledged need for culturally safe exercise-based interventions, such as pulmonary rehabilitation programs 
$(23,38)$, it was expected more studies would have been available for review, particularly in the grey literature where Indigenous health interventions and evaluations are more often published (62).

\section{Outcome of intervention}

The included study was a single arm intervention, rated as weak quality, that recruited Indigenous participants with COPD or ischaemic heart disease (IHD) or chronic heart failure (CHF) but did not provide outcomes specifically for participants with COPD. While there was only one study considered suitable for inclusion in this review, making generalisation difficult, the findings provide numerous points for consideration. The results of this study suggest that cardiopulmonary rehabilitation programs, delivered by an Aboriginal Community Controlled Health Service in Australia, have the potential to achieve clinically important improvements in exercise capacity, and a reduction in breathlessness and improvement in some domains of quality of life in Indigenous participants with COPD, IHD or CHF. The study stated important factors for success were strong internal executive leadership and staff commitment to deliver a sustainable program that was supported through core annual service funding. In this study, cardiopulmonary rehabilitation programs were provided through an Aboriginal Community Controlled Health Service, on their premises or at private practices of exercise physiologists/ physiotherapists. The process of assessing the cultural safety of these private practices by Indigenous study participants or local Indigenous communities was not reported and the effect of changing the program venue to a private practice was not explored. In future studies it would be important to evaluate participants' perspectives whether they felt the programs and program venues were culturally safe. Although the study aimed to explore the cost-effectiveness of the program, no data were provided. Such data would be important to support the economic case for providing Indigenous specific pulmonary rehabilitation programs.

Importantly, program participants and staff highlighted positive outcomes. Participants reported the value of 'being with your own community'. Such connectivity, through exercise-based interventions, has been shown to facilitate cultural and community connectivity (63). The importance of cultural identity and Indigenous community connectivity was highlighted as enhancing the outcomes of the study, similar to other studies in which Indigenous peoples participated $(63,64)$.

\section{Culturally-centred approaches}

The study highlighted the importance of culturally-centred approaches. The authors developed a holistic, integrated, and comprehensive intervention with a focus on primary and secondary prevention for Indigenous peoples with, or at risk of developing, respiratory or cardiac conditions. This approach reflects the values of the Australian Aboriginal Community Controlled Health Service and Indigenous peoples holistic construct of health (65). The holistic approach taken resulted in a general, rather than a diseasespecific rehabilitation program. The included study raises important questions about the value and impact of disease-specific programs and their alignment with holistic worldviews and beliefs of Indigenous peoples. However, evidence supporting the health benefits of general, rather than disease-specific rehabilitation programs for people with chronic lung disease is not yet well established (23). Whether future pulmonary rehabilitation programs for Indigenous peoples should include broader inclusion criteria with a primary and secondary prevention focus, and transition away from an individual organ or disease approach, as conducted in a recent Indigenous chronic disease study (66), remains debatable.

Learnings from published physical activity and cardiac rehabilitation programs for Indigenous peoples may be applicable to designing and delivering culturally safe pulmonary rehabilitation programs $(64,67-73)$. Cardiac rehabilitation literature has clear guides for health professionals to provide culturally safe programs with Indigenous staff leading exercise training classes (69). Additional learnings include approaching rehabilitation from a holistic and flexible primary and secondary prevention model (64), integrated with Indigenous community controlled health services and incorporating community and cultural aspects into the program's design, delivery and participant education (68). Examples of incorporating cultural aspects include, relating the distance participants cycled to the distance 'travelled' on a map denoting culturally important places and describing the country visited (74), providing gender specific programs in line with cultural traditions, the use of a buddy or mentoring system to encourage attendance and inclusion of cultural mentors (69).

\section{Indigenous engagement, Indigenous involvement, and Indigenous governance}

Indigenous engagement, involvement and governance 
supports the integration of cultural values, traditions and beliefs, while strengthening Indigenous decision-making, self-determination and capacity (75-79). Indigenous engagement was evident in the included study with Indigenous staff of the Aboriginal Community Controlled Health Service involved in the design of the intervention, which was based on national guidelines for rehabilitation programs. The described rehabilitation program's structure and content was similar to other programs implemented by mainstream health organisations for the general population with chronic disease $(23,80,81)$, which may not always be culturally safe for Indigenous peoples. Indigenous involvement on the research team was evident, with one of the three authors identified as Indigenous, enabling the application of an Indigenous lens to this Indigenous specific research. Using Indigenous research methodologies such as yarning circles (82) and involving Indigenous participants to validate the themes and findings would have strengthened the study. During program delivery, Indigenous involvement was evident mainly through AHWs who played a pivotal role in community engagement, due to existing relationships with community members and awareness of cultural values and knowledges (78). While it is important that trained health professionals, such as physiotherapists and exercise physiologists provide pulmonary rehabilitation exercise prescription and supervision, a role exists for AHWs to assist exercise training and the provision of education sessions in a culturally and clinically safe manner (69). Indigenous governance was evident in this study through the State Committee of the Tasmanian Aboriginal Centre and the Human Research Ethics Committee Tasmania Network granting ethics approval and an Aboriginal Community Controlled Service providing clinical and organisational governance. There was however, no mention of an Indigenous reference committee consisting of local community to ensure the intervention reflected and adhered to community values and beliefs (55).

The paper raises a valuable point of discussion, that Indigenous community controlled health services in Australia, New Zealand, Canada, and USA are uniquely positioned to deliver pulmonary rehabilitation programs specifically designed for Indigenous peoples. The successful provision of pulmonary rehabilitation programs for Indigenous peoples that are culturally safe, efficient and effective will require a clearer understanding of the barriers and enablers to such programs. While this review found limited literature for Indigenous specific exercised-based interventions, such as pulmonary rehabilitation programs, future programs should strive to incorporate similar Indigenous knowledges, values and beliefs, guided by strong Indigenous governance and methodologies. Continued research in this area should aim to have a greater influence on public policy and translation to practice, economic feasibility and scalability (55).

\section{Strengths}

The review was guided by a prospectively registered protocol, incorporated a relevant Indigenous theoretical framework, and actively engaged Indigenous scholars on the research team (DP Meharg and J Gilroy) to apply an Indigenous perspective.

\section{Limitations}

The review was limited due to the scarcity of publications relating to exercise-based interventions, such as pulmonary rehabilitation programs for Indigenous peoples with COPD. The lack of data meant that no meta-analyses could be performed and thus no conclusions or recommendations could be made regarding pulmonary rehabilitation programs for Indigenous adults with chronic lung disease, such as COPD.

\section{Conclusions}

This review highlights the paucity of peer-reviewed literature about exercise-based interventions, such as pulmonary rehabilitation programs for Indigenous adults with chronic lung disease living in Australia, New Zealand, Canada or USA. Despite the disproportionate impact of chronic lung disease on Indigenous peoples, published literature of exercise-based interventions for Indigenous peoples are almost non-existent. Additional research is required to address this important gap, guided by strong Indigenous governance, involvement and engagement.

\section{Acknowledgments}

We acknowledge Elaine Tam, Academic Liaison Librarian, Faculty of Medicine and Health, Academic Services, The University of Sydney Library for her technical support of database searches.

Funding: This work was supported by funding from the National Health \& Medical Research Council Australia, Global Alliance for Chronic Disease grant (1116081) to the 
University of Sydney.

\section{Footnotes}

Reporting Checklist: The authors have completed the PRIMSA reporting checklist. Available at http://dx.doi. org/10.21037/jtd-20-1904

Conflicts of Interest: All authors have completed the ICMJE uniform disclosure form (available at http://dx.doi. org/10.21037/jtd-20-1904). All authors report grants from National Health and Medical Research Council, Australia, during the conduct of the study. DPM is supported as a Fellow, Wingara Mura Leadership Program, University of Sydney. The authors have no other conflicts of interest to declare.

Ethical Statement: The authors are accountable for all aspects of the work in ensuring that questions related to the accuracy or integrity of any part of the work are appropriately investigated and resolved.

Open Access Statement: This is an Open Access article distributed in accordance with the Creative Commons Attribution-NonCommercial-NoDerivs 4.0 International License (CC BY-NC-ND 4.0), which permits the noncommercial replication and distribution of the article with the strict proviso that no changes or edits are made and the original work is properly cited (including links to both the formal publication through the relevant DOI and the license). See: https://creativecommons.org/licenses/by-nc-nd/4.0/.

\section{References}

1. Schofield T, Gilroy J. Indigeneity and health. In: Toni Schofield (eds). A Sociological Approach to Health Determinants. Melbourne: Cambridge University Press, 2015:99-122.

2. United Nations. State of the World's Indigenous Peoples: Indigenous Peoples' access to Health Services. United Nations, Information DoP, 2010. Report No. DPI/2551/ E-09-64058.

3. Australian Institute of Health and Welfare. Coronary heart disease and chronic obstructive pulmonary disease in Indigenous Australians. Canberra: AIHW, 2014.

4. Ministry of Health. Tatau Kahukura: M ori Health Chart Book 2015. 3rd ed. Wellington: Ministry of Health, 2015.

5. Ospina M, Voaklander D, Senthilselvan A, et al. Incidence and Prevalence of Chronic Obstructive Pulmonary Disease among Aboriginal Peoples in Alberta, Canada. PLoS One 2015;10:e0123204.

6. Basnayake TL, Morgan LC, Chang AB. The global burden of respiratory infections in indigenous children and adults: A review. Respirology 2017;22:1518-28.

7. Blackall SR, Hong JB, King P, et al. Bronchiectasis in indigenous and non indigenous residents of Australia and New Zealand. Respirology 2018;23:743-9.

8. Yang IA, Brown JL, George J, et al. COPD-X Australian and New Zealand guidelines for the diagnosis and management of chronic obstructive pulmonary disease: 2017 update. Med J Aust 2017;207:436-42.

9. Australian Bureau of Statistics. Causes of Death, Australia, 2017. Leading causes of death in Aboriginal and Torres Strait Islander People, 2017.

10. Australian Institute of Health and Welfare. Chronic obstructive pulmonary disease (COPD). Cat. no. ACM 35 Canberra: AIHW, 2019. Available online: https://www. aihw.gov.au/reports/chronic-respiratory-conditions/copd

11. Storme M, Semionov A, Assayag D, et al. Estimating the incidence of interstitial lung diseases in the Cree of Eeyou Istchee, northern Québec. PLoS One 2017;12:e0184548.

12. DiGiacomo M, Davidson PM, Abbott PA, et al. Smoking cessation in indigenous populations of Australia, New Zealand, Canada, and the United States: elements of effective interventions. Int J Environ Res Public Health 2011;8:388-410.

13. Senthilselvan A, Habbick BF. Increased asthma hospitalizations among registered Indian children and adults in Saskatchewan, 1970-1989. J Clin Epidemiol 1995;48:1277-83.

14. O'Grady KA, Hall K, Bell A, Chang A, Potter C. Review of respiratory diseases among Aboriginal and Torres Strait Islander children. Available online: http://healthbulletin. org.au/wp-content/uploads/2018/07/RespiratoryReview-2018.pdf

15. ACSQHC. Second Australian Atlas of Healthcare Variation. Contract No 1.1 Chronic obstructive pulmonary disease. Sydney: Australian Commission on Safety and Quality in Health Care, 2017.

16. GOLD 2019 global strategy for prevention, diagnosis and management of COPD. Available online: https://goldcopd. org/gold-reports/

17. Access Economics. Access economics pty limited for the australian lung foundation: Economic impact of COPD and cost-effective solutions 2008. Available online: http:// www.rnig.org.au/docs/EconomicImpactofCOPDandCost 
EffectiveSolutions-226.pdf

18. Brooke ME, Spiliopoulos N, Collins M. A review of the availability and cost effectiveness of chronic obstructive pulmonary disease (COPD) management interventions in rural Australia and New Zealand. Rural Remote Health 2017;17:4017.

19. Dang-Tan T, Ismaila A, Zhang $S$, et al. Clinical, humanistic, and economic burden of chronic obstructive pulmonary disease (COPD) in Canada: a systematic review. BMC Res Notes 2015;8:464.

20. Guarascio AJ, Ray SM, Finch CK, et al. The clinical and economic burden of chronic obstructive pulmonary disease in the USA. Clinicoecon Outcomes Res 2013;5:235-45.

21. O'Donnell DE, Aaron S, Bourbeau J, et al. Canadian Thoracic Society Recommendations for Management of Chronic Obstructive Pulmonary Disease - 2007 Update. Can Respir J 2007;14:5B-32B.

22. Qaseem A, Wilt TJ, Weinberger SE, et al. Diagnosis and management of stable chronic obstructive pulmonary disease: a clinical practice guideline update from the American College of Physicians, American College of Chest Physicians, American Thoracic Society, and European Respiratory Society. Ann Intern Med 2011;155:179-91.

23. Alison JA, McKeough ZJ, Johnston K, et al. Australian and New Zealand Pulmonary Rehabilitation Guidelines. Respirology 2017;22:800-19.

24. McCarthy B, Casey D, Devane D, et al. Pulmonary rehabilitation for chronic obstructive pulmonary disease. Cochrane Database Syst Rev 2015;(2):CD003793.

25. Puhan MA, Gimeno-Santos E, Cates CJ, et al. Pulmonary rehabilitation following exacerbations of chronic obstructive pulmonary disease. Cochrane Database Syst Rev 2016;12:CD005305.

26. Dowman L, Hill CJ, Holland AE. Pulmonary rehabilitation for interstitial lung disease. Cochrane Database Syst Rev 2014;(10):CD006322.

27. Lee AL, Cecins N, Hill CJ, et al. The effects of pulmonary rehabilitation in patients with non-cystic fibrosis bronchiectasis: protocol for a randomised controlled trial. BMC Pulm Med 2010;10:5.

28. Spruit MA, Singh SJ, Garvey C, et al. An official American Thoracic Society/European Respiratory Society statement: key concepts and advances in pulmonary rehabilitation. Am J Respir Crit Care Med 2013;188:e13-64.

29. Vestbo J, Hurd SS, Agustí AG, et al. Global strategy for the diagnosis, management, and prevention of chronic obstructive pulmonary disease: GOLD executive summary.
Am J Respir Crit Care Med 2013;187:347-65.

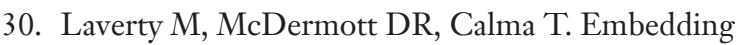
cultural safety in Australia's main health care standards. Med J Aust 2017;207:15-6.

31. Australian Commission on Safety and Quality in Health Care. Health literacy: taking action to improve safety and quality. Sydney: Australian Commission on Safety and Quality in Health Care, 2014.

32. Willging CE, Sommerfeld DH, Jaramillo ET, et al. "Improving Native American elder access to and use of health care through effective health system navigation". BMC Health Serv Res 2018;18:464.

33. Paradies Y, Harris R, Anderson I. The impact of racism on indigenous health in Australia and Aotearoa: towards a research agenda. Casuarina, N.T: Cooperative Research Centre for Aboriginal Health, 2008.

34. Hole RD, Evans M, Berg LD, et al. Visibility and Voice: Aboriginal People Experience Culturally Safe and Unsafe Health Care. Qual Health Res 2015;25:1662-74.

35. Wepa D. Cultural safety in Aotearoa New Zealand. Auckland, New Zealand: Pearson Education New Zealand, 2005.

36. Papps E, Ramsden I. Cultural safety in nursing: the New Zealand experience. Int J Qual Health Care 1996;8:491-7.

37. Richardson S. Aoteaoroa/New Zealand nursing: from eugenics to cultural safety. Nursing Inquiry 2004;11:35-42.

38. Levack WMM, Jones B, Grainger R, et al. Whakawhanaungatanga: the importance of culturally meaningful connections to improve uptake of pulmonary rehabilitation by Maori with COPD--a qualitative study. Int J Chron Obstruct Pulmon Dis 2016;11:489.

39. Davey M, Moore W, Walters J. Tasmanian Aborigines step up to health: evaluation of a cardiopulmonary rehabilitation and secondary prevention program. BMC Health Serv Res 2014;14:349.

40. Keating A, Lee A, Holland AE. What prevents people with chronic obstructive pulmonary disease from attending pulmonary rehabilitation? A systematic review. Chron Respir Dis 2011;8:89-99.

41. National Aboriginal Health Strategy Working Party. National Aboriginal Health Strategy. Canberra, 1989.

42. Panaretto KS, Wenitong $M$, Button $S$, et al. Aboriginal community controlled health services: leading the way in primary care. Med J Aust 2014;200:649-52.

43. Dwyer D, Silburn K, Wilson G. National Strategies for Improving Indigenous Health and Health Care: Aboriginal and Torres Strait Islander Primary Health Care Review: Consultant Report No 1. La Trobe University, 2004. 
44. Gifford H, Cvitanovic L, Boulton A, et al. Constructing prevention programmes with a Māori health service provider view. Kōtuitui 2017;12:165-78.

45. Roubideaux Y. Perspectives on American Indian health. Am J Public Health 2002;92:1401-3.

46. Harfield S, Davy C, Kite E, et al. Characteristics of Indigenous primary health care models of service delivery: a scoping review protocol. JBI Database System Rev Implement Rep 2015;13:43-51.

47. Richmond CAM, Cook C. Creating conditions for Canadian aboriginal health equity: the promise of healthy public policy. Public Health Rev 2016;37:2.

48. Moher D, Liberati A, Tetzlaff J, et al. Preferred reporting items for systematic reviews and meta-analyses: the PRISMA statement. Ann Intern Med 2009;151:264-9.

49. Smith LT. Decolonizing methodologies: research and indigenous peoples 2nd ed. London: Zed Books, 2012.

50. Kalinin D, Gilroy J, Pinckham S. The needs of carers of Aboriginal and Torres Strait Islander children and young people in foster care in Australia: A systematic literature review, July 2018. Sydney, Australia: Macquarie University and The University of Sydney, 2018.

51. Gilroy J, Donelly M, Colmar S, et al. Conceptual framework for policy and research development with Indigenous people with disabilities. Australian Aboriginal Studies 2013;(2):42-58.

52. Effective Public Health Practice Project. Quality Assessment Tool for Quantitative Studies, 2009. Available online: https://www.ephpp.ca/quality-assessment-tool-forquantitative-studies/?doing_wp_cron=1605485859.741486 0725402832031250

53. Pelletier CA, Smith-Forrester J, Klassen-Ross T. A systematic review of physical activity interventions to improve physical fitness and health outcomes among Indigenous adults living in Canada. Prev Med Rep 2017;8:242-9.

54. Godin K, Leatherdale ST, Elton-Marshall T. A systematic review of the effectiveness of school-based obesity prevention programmes for First Nations, Inuit and Métis youth in Canada. Clin Obes 2015;5:103-15.

55. Sushames A, van Uffelen JGZ, Gebel K. Do physical activity interventions in Indigenous people in Australia and New Zealand improve activity levels and health outcomes? A systematic review. Int J Behav Nutr Phys Act 2016;13:129.

56. Deeks JJ, Dinnes J, D'Amico R, et al. Evaluating nonrandomised intervention studies. Health Technol Assess 2003; 7:iii-173.
57. Jackson N, Waters E. Criteria for the systematic review of health promotion and public health interventions. Health Promot Int 2005;20:367-74.

58. Armijo-Olivo S, Stiles CR, Hagen NA, et al. Assessment of study quality for systematic reviews: a comparison of the Cochrane Collaboration Risk of Bias Tool and the Effective Public Health Practice Project Quality Assessment Tool: methodological research. J Eval Clin Pract 2012;18:12-8.

59. Ryan R. Cochrane Consumers and Communication Review Group. 'Cochrane Consumers and Communication Review Group: data synthesis and analysis', 2019. Available online: https://cccrg.cochrane.org/

60. Thomas BH, Ciliska D, Dobbins M, et al. A Process for Systematically Reviewing the Literature: Providing the Research Evidence for Public Health Nursing Interventions. Worldviews Evid Based Nurs 2004;1:176-84.

61. Holland AE, Spruit MA, Troosters T, et al. An official European Respiratory Society/American Thoracic Society technical standard: field walking tests in chronic respiratory disease. Eur Respir J 2014;44:1428-46.

62. Gomersall JS, Canuto K, Aromataris E, et al. Systematic review to inform prevention and management of chronic disease for Indigenous Australians: overview and priorities. Aust N Z J Public Health 2016;40:22-9.

63. Tang K, Jardine C. Our Way of Life: Importance of Indigenous Culture and Tradition to Physical Activity Practices. Int J Indig Health 2016;11:211-27.

64. Field PE, Franklin RC, Barker RN, et al. Cardiac rehabilitation services for people in rural and remote areas: an integrative literature review. Rural Remote Health 2018;18:4738.

65. Aboriginal Health \& Medical Research Council. Aboriginal communities improving Aboriginal health: An evidence review on the contribution of Aboriginal Community Controlled Health Services to improving Aboriginal health. Sydney: AH\&MRC, 2015.

66. Hu J, Basit T, Nelson A, et al. Changes in exercise capacity and anthropometric measures after Work It Out-a holistic chronic disease self-management program for urban Aboriginal and Torres Strait Islander people. Public Health 2019;174:49-55.

67. Lavallée L. Physical Activity and Healing through the Medicine Wheel. Social Work, 2007;5.

68. Digiacomo ML, Thompson SC, Smith JS, et al. 'I don't know why they don't come': barriers to participation in cardiac rehabilitation. Aust Health Rev 2010;34:452-7.

69. Hamilton S, Mills B, McRae S, et al. Cardiac 
Rehabilitation for Aboriginal and Torres Strait Islander people in Western Australia. BMC Cardiovasc Disord 2016;16:150.

70. Hayman NE, Wenitong M, Zangger JA, et al. Strengthening cardiac rehabilitation and secondary prevention for Aboriginal and Torres Strait Islander peoples. The Med J Aust 2006;184:485.

71. Taylor KP, Smith JS, Dimer L, et al. "You're always hearing about the stats ... death happens so often": new perspectives on barriers to Aboriginal participation in cardiac rehabilitation. Med J Aust 2010;192:602.

72. Thompson SC, DiGiacomo ML, Smith JS, et al. Are the processes recommended by the NHMRC for improving Cardiac Rehabilitation (CR) for Aboriginal and Torres Strait Islander people being implemented? An assessment of CR Services across Western Australia. Aust New Zealand Health Policy 2009;6:29.

73. Walsh WF, Kangaharan N. Cardiac care for Indigenous Australians: practical considerations from a clinical perspective. Med J Aust 2017;207:40-5.

74. Dimer L, Dowling T, Jones J, et al. Build it and they will come: outcomes from a successful cardiac rehabilitation program at an Aboriginal Medical Service. Aust Health Rev 2013;37:79-82.

75. Tsey K, McCalman J, Bainbridge R, et al. Strengthening

Cite this article as: Meharg DP, Gwynne K, Gilroy J, Alison JA. Exercise-based interventions for Indigenous adults with chronic lung disease in Australia, Canada, New Zealand, and USA: a systematic review. J Thorac Dis 2020;12(12):7442-7453. doi: $10.21037 /$ jtd-20-1904 organisational capacity to improve Indigenous Australian community governance: A two-way approach. Int Rev Social Sci Humanities 2012;2:162-75.

76. Gwynne K, Jeffries T, Lincoln M. Improving the efficacy of healthcare services for Aboriginal Australians. Aust Health Rev 2019;43:314-22.

77. Durey A, McEvoy S, Swift-Otero V, et al. Improving healthcare for Aboriginal Australians through effective engagement between community and health services. BMC Health Serv Res 2016;16:224.

78. Harding T, Oetzel J. Implementation effectiveness of health interventions for indigenous communities: A systematic review. Implement Sci 2019;14:76.

79. Parter C, Wilson S, Hartz DL. The Closing the Gap (CTG) Refresh: Should Aboriginal and Torres Strait Islander culture be incorporated in the CTG framework? How? Aust N Z J Public Health 2019;43:5.

80. Jenkins S, Hill K, Cecins NM. State of the art: How to set up a pulmonary rehabilitation program. Melbourne, Australia: Blackwell Publishing Asia, 2010:1157-73.

81. Alison JA. The Pulmonary Rehabilitation Toolkit. In Brisbane: Australian Lung Foundation, 2009.

82. Ryder C, Mackean T, Coombes J, et al. Indigenous research methodology - weaving a research interface. Int J Soc Res Methodol 2020;23:255-67. 